C 2020 Universidad Nacional Autónoma de México, Facultad de Estudios Superiores Zaragoza.

Este es un artículo Open Access bajo la licencia CC BY-NC-ND (http://creativecommons.org/licenses/by-nc-nd/4.0/).

TIP Revista Especializada en Ciencias Químico-Biológicas, 23: 1-9, 2020.

https://doi.org/10.22201/fesz.23958723e.2020.0.239

\title{
Alteraciones del olfato en la obesidad
}

\author{
Marco Guarneros ${ }^{1}$, Margarita Martínez-Gómez ${ }^{1,2}$ \\ y Lourdes Arteaga ${ }^{1 *}$ \\ ${ }^{1}$ Posgrado en Ciencias Biológicas, Centro Tlaxcala Biología de la Conducta, \\ Universidad Autónoma de Tlaxcala, Km. 1.5 carretera Tlaxcala/Puebla, Tlaxcala, \\ 90070, México. ${ }^{2}$ Instituto de Investigaciones Biomédicas, UNAM, México. \\ E-mail: *mariadelourdes.arteagac@uatx.mx, ORCID 0000-0002-9678-9442
}

\begin{abstract}
RESUMEN
Aun cuando el sentido del olfato es fundamental para la percepción de los alimentos, su relación con la obesidad ha sido poco investigada. Este artículo es una revisión de la literatura sobre esa relación. La evidencia actual tanto en animales como en humanos apoya la hipótesis de que el olfato está alterado en esta condición. Estudios recientes señalan que los individuos obesos podrían tener una mayor sensibilidad a los olores de alimentos altos en calorías, y menor para los de bajo valor calórico incluidos los no asociados con alimentos. El estudio de la percepción quimiosensorial es un área prometedora para avanzar en el entendimiento de los mecanismos involucrados en la obesidad.
\end{abstract}

Palabras clave: humanos, obesidad, revisión, función olfatoria, sentido del olfato.

\section{Olfactory alterations in obesity}

\begin{abstract}
Although the sense of smell is fundamental for the perception of food, its relationship with obesity has not been sufficiently researched. This article is a review of the literature of the relationship between olfactory function and obesity. Current evidence from animal and human studies supports the hypothesis that olfactory function is altered in obesity. Recent research indicates that individuals with obesity could have a higher sensitivity to and preference for odorant stimuli associated with energy-dense foods, and lower sensitivity to odors of foods with low caloric value and non-food odors. The study of chemosensory perception is a promising area for advancing in the understanding of the mechanisms involved in obesity.
\end{abstract}

Keywords: humans, obesity, review, olfactory function, sense of smell. 


\section{INTRODUCCIÓN}

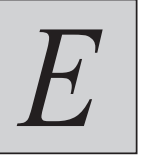

n las últimas décadas, la obesidad se ha convertido en una epidemia global que afecta la salud y el bienestar del ser humano. Mientras que este problema es especialmente grave en países desarrollados, su prevalencia se está incrementando rápidamente en muchos países en desarrollo. En América Latina y el Caribe, la obesidad es un problema en aumento que tiene un impacto mayor en las mujeres y una tendencia al alza en niños y niñas, de acuerdo con la Organización de las Naciones Unidas para la Alimentación y la Agricultura (FAO), y la Organización Panamericana de la Salud. Según un informe reciente, más del $23 \%$ de los habitantes adultos latinoamericanos presentan obesidad y esta proporción podría incrementarse al $30 \%$ para el año 2030. La evidencia señala que el cambio en los patrones alimentarios, impulsado por el crecimiento económico, la urbanización y la integración de la región en los mercados internacionales, han reducido el consumo de alimentos tradicionales y aumentado el consumo de productos ultra procesados (FAO, OPS, WFP \& UNICEF, 2018; Popkin, Aldair \& Ng, 2012).

Un aspecto que ha recibido poca atención es el papel que juega el sentido del olfato en la obesidad, lo que sorprende si consideramos que las propiedades sensoriales son esenciales en la evaluación de los alimentos y en la preferencia alimenticia, y que el olfato determina en gran medida el sabor de los alimentos (Stevenson, 2010). También se ha encontrado que la disfunción olfatoria tiene efectos en la conducta alimentaria y que se asocia con cambios en el peso corporal, así como con la pérdida de placer por la comida y la pérdida del apetito (Hummel \& Nordin, 2005; Aschenbrenner et al., 2008; Stevenson, 2010).

Aunque la etiología de la obesidad parece ser multifactorial, un factor importante es la alimentación moderna, que alienta el consumo excesivo de alimentos altos en calorías y con un bajo contenido de nutrientes. La posibilidad de que el olfato esté alterado en la obesidad surge porque en las últimas décadas han aumentado significativamente las cualidades sensoriales y la disponibilidad de los alimentos, mientras que las señales de saciedad producidas por la distención estomacal y hormonas han permanecido sin cambios. Esto resulta en un incremento neto promedio en el valor de la recompensa y la palatabilidad del alimento, lo que anula las señales de saciedad y contribuye a la tendencia de comer demasiado (Rolls, 2011; Peng, Coutts, Wang \& Cakmak, 2019). De acuerdo con Rolls (2011), las señales internas asociadas a la saciedad - como la distención gástrica y el consumo de glucosa - evolucionaron como indicaciones que conducen al individuo a dejar de comer después de haber ingerido una cierta cantidad de alimento. Sin embargo, estas señales de saciedad se ven rebasadas ante la mayor palatabilidad y características sensoriales de los alimentos modernos, los cuales favorecen la sobreingesta. Además, algunos individuos podrían ser particularmente sensibles a los efectos de los estímulos visuales de la publicidad sobre los sistemas cerebrales asociados a la recompensa por alimentos. La representación de un alimento placentero en la corteza orbitofrontal - en ausencia de señales de saciedad - produce una respuesta en áreas como el estriado y el giro cingulado, que desencadena la conducta alimentaria. Es importante avanzar en el entendimiento acerca de las reglas que utiliza el cerebro para producir la representación del agrado por el alimento y cómo el sistema es modulado por su ingesta y por la saciedad. Este entendimiento, además de cómo los factores sensoriales pueden ser diseñados y controlados para no anular las señales de saciedad, son áreas de investigación importantes en el entendimiento, prevención y tratamiento de la obesidad (Rolls, 2011).

La alimentación moderna también provee nuevos ingredientes, cuyos efectos en la salud y en la percepción de los alimentos en gran medida se ignoran. Por ejemplo, algunos edulcorantes no calóricos podrían influir en las señales gástricas de distintas maneras. Un estudio sobre la regulación del apetito reveló que la administración de aspartame una hora antes de empezar a comer, reduce significativamente la ingesta de alimentos (Rogers, Burley, Alikhanizadeh \& Blundell, 1995).

El objetivo del presente estudio es revisar la literatura existente sobre el papel que juega el olfato en la obesidad y discutir cómo el olfato contribuye en la compleja etiopatología de la obesidad.

\section{PERCEPCIÓN SENSORIAL EN LA OBESIDAD}

Los sentidos del gusto y de la vista han sido las modalidades sensoriales más exploradas en relación con la obesidad. En el caso del sentido del gusto, las investigaciones son inconsistentes hasta ahora, ya que algunos estudios no encuentran diferencias en las sensibilidades del gusto entre grupos de distinto peso (Tucker, Edlinger, Craig \& Mattes, 2014; Bartoshuk, Duffy, Hayes, Moskowitz \& Snyder, 2006), mientras que otros estudios sugieren mayores o menores sensibilidades asociadas con la obesidad (Bartoshuk et al., 2006; Overberg, Hummel, Krude \& Wiegand, 2012; Liu, Archer, Duesing, Hannan \& Keast, 2016). Estas inconsistencias pueden reflejar los hallazgos discrepantes para las diferentes percepciones gustativas. Una revisión de la literatura sugiere que la sensibilidad del gusto está disminuida en individuos con obesidad pero sólo para la percepción de estímulos ácidos y amargos, ya que se incrementa para estímulos salados y se mantiene sin cambios para la percepción de lo dulce (Donaldson, Bennet, Baic \& Melichar, 2009). Otro estudio encontró que los sujetos con obesidad son más sensibles que los individuos delgados a los estímulos dulces y salados (Hardikar, Höchenberger, Villringer \& Ohla, 2017). También se ha reportado que las mujeres con obesidad tienen menor sensibilidad gustativa para el glutamato de sodio y que prefieren concentraciones más altas de este estímulo en comparación con mujeres de peso normal (Pepino, Finkbeiner, Beauchamp \& Mennella, 2010). 
En el caso del sentido de la vista, estudios conductuales y de seguimiento ocular (eye-tracking) han mostrado que individuos con sobrepeso tienen un sesgo atencional hacia las pistas asociadas a alimentos que no se observa en individuos de peso normal (Wang, Cakmak \& Peng, 2018; Hendrikse et al., 2015). Conductualmente, este sesgo de atención diferencial en distintos grupos de peso ha sido explicado por medio de la teoría de la sensibilización al incentivo (Robinson \& Berridge, 2008; Yokum, Ng \& Stice, 2011). Esta teoría ha sido adaptada de la investigación en adicciones y propone que los individuos con adicción tienen mayores sensibilidades hacia estímulos asociados a recompensas (Robinson \& Berridge, 2008). Neurológicamente, estudios de neuroimagen han confirmado que al visualizar pistas alimenticias, los individuos con sobrepeso exhiben una mayor activación que individuos de peso normal en regiones cerebrales relacionadas con atención y recompensa alimenticia, como la ínsula anterior, la corteza lateral orbitofrontal, la corteza prefrontal ventrolateral y el lóbulo parietal superior. Por otro lado, los autores sugieren que los individuos jóvenes que muestran altos niveles de activación en el circuito de recompensa durante la exposición a pistas alimenticias podrían estar en riesgo de desarrollar sobrepeso (Yokum et al., 2011).

\section{EVIDENCIA DERIVADA DE ESTUDIOS EN ANIMALES}

De los sentidos de percepción, el olfato es el menos entendido en términos de su relación con la obesidad. Parcialmente, esta brecha de conocimiento refleja la complejidad relativa del sistema olfatorio de los mamíferos. Las moléculas odorantes del medio ambiente son reconocidas por los receptores de neuronas de primer orden en la mucosa nasal, que envían la señal hacia el bulbo olfatorio (Figura 1). Esta información olfatoria es transportada al núcleo olfatorio, la corteza piriforme y la amígdala, que en conjunto componen a la corteza olfatoria primaria. Más allá de la corteza olfatoria primaria, proyecciones de mayor orden de información olfatoria convergen en la corteza orbitofrontal, la ínsula agranular, el tálamo, hipotálamo, ganglios basales e hipocampo. La convergencia de información sensorial gustativa, olfatoria, táctil y visual del alimento ocurre en la corteza orbitofrontal para construir una representación del sabor del alimento. La corteza orbitofrontal es la región donde están representados el sabor, el agrado y la palatabilidad del alimento. En conjunto, esta compleja red neural determina el desempeño olfatorio de un individuo y regula las conductas guiadas por olores (Firestein, 2001; Lledo, Gheusi \& Vincent, 2005).

A inicios de la década de 1970, Pager, Giachetti, Holley \& Le Magnen (1972) y Giachetti, MacLeod \& LeMagnen (1970) fueron los primeros en reportar que la actividad de las células mitrales del bulbo olfatorio pudiera estar perturbada en ratas en ayuno, en comparación con ratas saciadas, en respuesta a olores de alimentos. Estos autores pioneros dedujeron correctamente que la actividad mitral es conducida por algunos factores relacionados con el estado metabólico (Palouzier-Paulignan

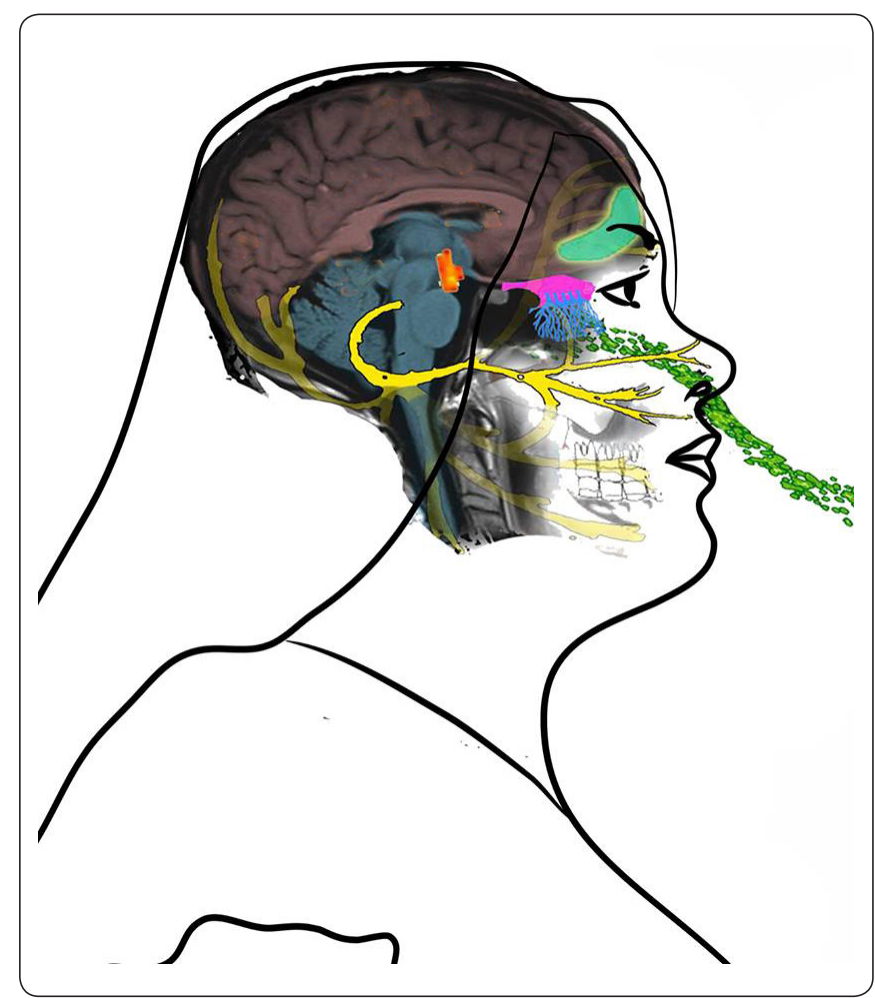

Figura 1. Ilustración de la señalización olfatoria. Las moléculas odorantes (verde oscuro) alcanzan el epitelio olfatorio (azul) al fondo de la cavidad nasal, en donde se encuentran los receptores olfatorios. La señal viaja al bulbo olfatorio (rosa) y de ahí a otras regiones como el hipotálamo (naranja), relacionado con el hambre y la conducta alimentaria. La corteza orbitofrontal (verde) se relaciona con la percepción consciente de los olores. El nervio trigémino (amarillo) inerva la cavidad nasal y media sensaciones como la frescura. Modificada de Guarneros \& Hudson, 2016.

et al., 2012). Recientemente, Apelbaum \& Chaput (2003) utilizaron un paradigma de alimentación para demostrar que el acceso diferencial al alimento puede causar un decremento drástico en la actividad de las células mitrales. Utilizando registros individuales de células mitrales, descubrieron que el aumento en la tasa de descarga de la célula mitral en respuesta al ayuno es menor en ratas habituadas a un régimen diario de alimento restringido durante 15 días, en comparación con ratas que ayunaron por primera vez. El bulbo olfatorio recibe proyecciones orexinérgicas del hipotálamo lateral, conocido por su función en el control del consumo de alimentos y el peso corporal. Las orexinas, involucradas en el control de la alimentación, son moduladoras de la reactividad a olores del bulbo olfatorio. De modo que cambios en el régimen alimenticio (por ejemplo, el cambio de largo plazo de tres comidas a una sola al día) pueden influir en el bulbo olfatorio por medio del sistema orexinérgico. Los autores concluyen que el bulbo olfatorio parece ser muy sensible a los cambios del estado interno del organismo y a las modificaciones ambientales (Apelbaum \& Chaput, 2003). 
Otro estudio mostró que al suprimir las neuronas sensoriales olfatorias de ratones, estos se hacen resistentes a la obesidad inducida por dieta (Riera et al., 2017). Por otro lado, en ese mismo estudio se mostró que la pérdida aguda de la percepción olfatoria después de la aparición de la obesidad no sólo abrogó una mayor ganancia de peso sino que además redujo la grasa corporal y la resistencia a la insulina. Los autores encontraron que la reducción del olfato estimula la actividad simpática, lo que resulta en activación de los adipocitos que promueven la lipólisis. En sentido inverso, la supresión del receptor IGF1 aumenta el desempeño olfatorio en ratones, y esto lleva a un incremento en la adiposidad y en la resistencia a la insulina. Los hallazgos de este estudio revelan una función bidireccional del sistema olfatorio que controla la homeostasis en respuesta a las señales sensoriales y hormonales.

La amígdala es una estructura que parece ligar el sentido del olfato con la ingesta de alimentos, ya que se ha observado que lesiones en el área medial posterodorsal de la amígdala inducen hiperfagia y obesidad en gatos, perros y monos. Con la convergencia de proyecciones provenientes del bulbo olfatorio, la amígdala posterodorsal posiblemente sea un punto nodal en el cual los estímulos olfatorios y neuroendocrinos son integrados para afectar la conducta alimentaria (King, 2006) (Figura 2).

Un estudio en la mosca de la fruta (Drosophila melanogaster) indicó que una dieta alta en grasa reduce la longevidad y las habilidades motoras y olfatorias (Jung, Kim, Han \& Kwon, 2018). El estudio evaluó la sensibilidad olfatoria en moscas alimentadas con una dieta alta en grasa. Se observó una reducción en la sensibilidad a 8 de los 10 odorantes utilizados, mientras que la respuesta se incrementó para uno de los estímulos (benzaldehído). Estos cambios conductuales estuvieron acompañados por un decremento en la expresión de un gen (DmOrco) que codifica para un receptor olfatorio en un órgano olfatorio periférico, lo cual sugiere que este gen, cuya expresión es influenciada por la grasa en la dieta, podría estar involucrado en la acción de señales metabólicas y sensoriales. Los autores sugieren que la sensibilidad olfatoria está parcialmente mediada por rutas asociadas al metabolismo de nutrientes (Jung et al., 2018).

EVALUACIÓN DE LA FUNCIÓN OLFATORIA EN HUMANOS Se han utilizado diversas metodologías para investigar las funciones que realiza este sentido. Una de las metodologías

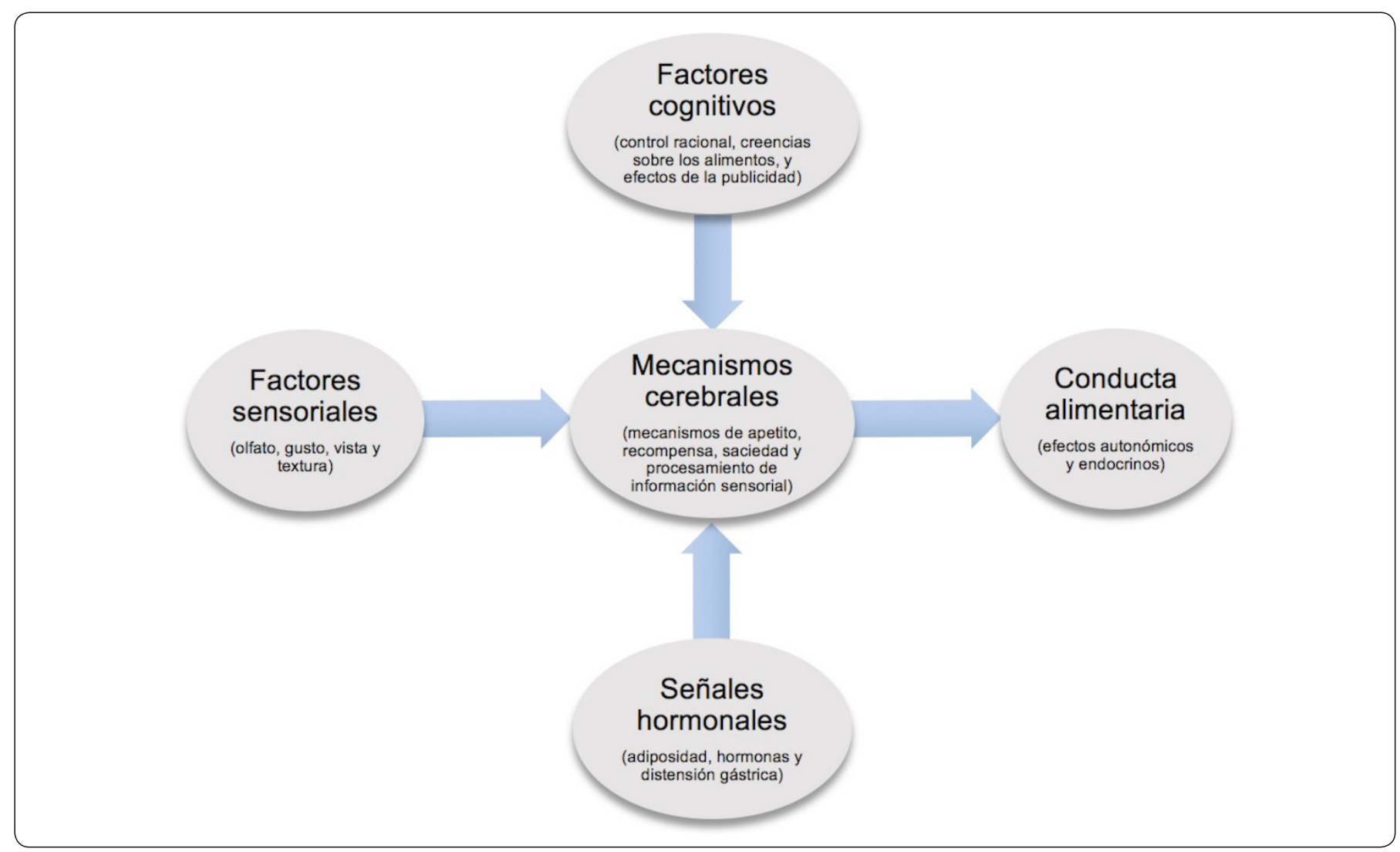

Figura 2. Diagrama de la interacción entre factores sensoriales y señales de saciedad en la corteza orbitofrontal para determinar el valor hedónico de un alimento, lo cual conduce al apetito y al acto de comer. Los factores cognitivos y atencionales modulan directamente el sistema de recompensa en el cerebro (Modificado de Rolls, 2011). 
más usadas actualmente es el kit de pruebas del olfato Sniffin' Sticks (Hummel, Sekinger, Wolf, Pauli \& Kobal, 1997), que mide tres aspectos de la función olfatoria: umbral, discriminación e identificación.

- Umbral: la prueba de umbral permite determinar la concentración mínima de un odorante a la que un sujeto es capaz de detectarlo. En este tipo de pruebas se le presentan al paciente concentraciones ascendentes de un odorante, comúnmente feniletanol (olor a rosa) o n-butanol (olor con cierta similitud a plátano, según algunas descripciones).

- Discriminación: se refiere a la capacidad que tiene el individuo de notar que un odorante es distinto de otro. La prueba de discriminación de los Sniffin'Sticks se compone de 16 tareas distintas, en cada una de las cuales el sujeto es presentado con tres plumones en orden aleatorio, dos de ellos con un mismo odorante. La tarea del sujeto consiste en decir cuál de los tres estímulos presentados es distinto de los otros dos.

- Identificación: en esta prueba el individuo debe oler consecutivamente 16 olores de la vida diaria, y la tarea consiste en que nombre correctamente cada estímulo con ayuda de una lista de opciones.

Además de la prueba de Sniffin' Sticks, se han usado otras para medir el olfato. Una de ellas es la conocida como UPSIT (University of Pennsylvania Smell Identification Test) (Doty, Shaman, Kimmelman \& Dann, 1984) de identificación de olores, ampliamente utilizada en investigación científica. Otra técnica, utilizada en un estudio sobre la relación entre la obesidad y el olfato (Simchen, Koebnick, Hoyer, Issanchou \& Zunft, 2006), es la prueba ETOC (European test of olfactory capabilities) que mide el umbral olfatorio y la identificación de olores (Thomas-Danguin et al., 2003). En otro estudio se utiliza una metodología pionera conocida como olfatometría de Elsberg Levy (Pruszewicz, 1965), que al igual que la anterior mide el umbral olfatorio y la identificación de olores.

De las pruebas mencionadas, la de Sniffin'Sticks es la única estandarizada y validada que mide el umbral, discriminación e identificación, y que ha sido utilizada en varios estudios para medir la capacidad olfatoria de la población general (Guarneros, Hudson, López-Palacios \& Drucker-Colín, 2015), incluida la pediátrica (Schriever et al., 2018), así como para caracterizar el olfato de pacientes con distintas patologías (Marin et al., 2018).

\section{EVIDENCIA ACTUAL DE ESTUDIOS EN HUMANOS}

Si bien se sabe que el olfato juega un papel fundamental en el consumo de alimentos (Yeomans, 2006), no está claro si las habilidades olfatorias están ligadas a la adiposidad a través de influencias directas sobre la conducta alimentaria. En la última década un número creciente de estudios ha intentado dar respuesta a esta cuestión investigando las habilidades olfatorias en grupos de distinto peso corporal (Islam et al., 2015; Peng et al., 2019).

En el año 2000, en una investigación sobre la relación entre olfato y obesidad en niños de 10 a 16 años, se determinó por medio del olfatómetro de Elsberg-Levy que los niños con obesidad tenían umbrales de detección olfatoria reducidos (Obrebowski, Obrebowska-Karsznia \& Gawlinski, 2000). Posteriormente, una investigación en adultos (Richardson, Vander Woude, Sudan, Thompson \& Leopold, 2004) indicó que la disfunción olfatoria podría ser un factor que contribuye con el progreso de la obesidad. En ese estudio (Richardson et al., 2004), por medio de una adaptación de la prueba UPSIT, se investigó la relación entre obesidad mórbida y el olfato en una muestra de 101 personas, y se encontró que los participantes con un índice de masa corporal $>45 \mathrm{~kg} / \mathrm{m}^{2}$ tienen mayor probabilidad de mostrar disfunción olfatoria que los participantes con valores más bajos de masa corporal. En un estudio similar (Simchen et al., 2006) se investigó la relación entre peso corporal y las funciones olfatorias de umbral e identificación, así como la percepción de las cualidades gustativas (salado, dulce, ácido y amargo) por medio de soluciones acuosas. En este estudio se utilizó la prueba llamada ETOC (Thomas-Danguin et al., 2001). La muestra total en este estudio fue de 313 y fue dividida en dos grupos de edades: 65 años de edad o más, y el grupo de 20 a 64 años de edad. Los puntajes para la detección e identificación de olores fueron menores en los participantes con un índice de masa corporal $\geq 28 \mathrm{~kg} / \mathrm{m}^{2}$ en el grupo menor de 65 años. Sin embargo, en el grupo mayor e igual de 65 años, los puntajes fueron mayores en participantes con un índice de masa corporal $\geq 28 \mathrm{~kg} / \mathrm{m}^{2}$ que en participantes con un índice de masa corporal $<28 \mathrm{~kg} / \mathrm{m}^{2}$. El estudio concluyó que la relación entre las capacidades sensoriales y el peso corporal depende de la edad, ya que en comparación con sujetos con sobrepeso, las capacidades sensoriales de quienes tienen un peso normal parecen ser mayores en personas menores de 65 años y menores después de los 65 años de edad.

La relación entre diabetes y función olfatoria fue el tema de investigación de un estudio (Gouveri et al. 2014). Con una muestra de 154 adultos, de los cuales el $77 \%$ tenían diabetes mellitus tipo 2, los autores encontraron que la diabetes mellitus tipo 2, la hipertensión y la hiperlipidemia están asociadas con bajos puntajes olfatorios en la prueba de Sniffin 'Sticks (umbral, discriminación, e identificación).

Recientemente, otro estudio investigó la relación entre peso corporal, percepción olfatoria y gustativa (Skrandies \& Zschieschang, 2015). En este estudio 66 sujetos saludables (20-56 años de edad) fueron evaluados por medio de la prueba de Sniffin'Sticks (umbral, discriminación e identificación) y una más para la función del gusto (umbral para dulce, ácido, salado y amargo). Se encontró una influencia significativa del 
índice de masa corporal en el umbral olfatorio. La influencia fue más alta en sujetos con mayor índice de masa corporal. En esta misma línea, se encontró que a mayor índice de masa corporal, la sensibilidad para el gusto "salado" disminuye, ya que el umbral de detección para este estímulo aumenta (se requieren mayores concentraciones para generar una respuesta en el individuo). Estos resultados indican que el peso corporal influye en la percepción gustatoria y olfatoria en adultos saludables. Los autores concluyen que los incrementos en el índice de masa corporal están asociados con un decremento en la sensibilidad del olfato y en la percepción de lo salado (Skrandies \& Zschieschang, 2015).

Un estudio más sobre el tema es el de Fernández-Aranda et al. (2016), quienes utilizaron los Sniffin' Sticks para analizar las tres variables del olfato en una muestra de sujetos con obesidad, individuos con anorexia e individuos controles. El grupo con obesidad tuvo puntajes significativamente más bajos en la prueba de umbral que un grupo de controles de la misma edad y que los pacientes con anorexia (quienes eran en promedio más jóvenes). Los hallazgos fueron similares para la identificación olfatoria, ya que en el grupo de personas con obesidad $54.3 \%$ de los sujetos tuvieron puntajes indicativos de hiposmia (reducción parcial de la capacidad olfatoria), mientras que sólo el $6.4 \%$ de los sujetos con anorexia resultaron hipósmicos. Los resultados muestran que la capacidad olfatoria de los individuos con obesidad está significativamente disminuida. Cabe mencionar que en este estudio no se encontraron diferencias entre los grupos para la percepción del gusto.

En 2017, Fernández-García y colaboradores analizaron las diferencias en las capacidades olfatorias y gustativas en mujeres con diferentes pesos corporales $(n=179)$, incluido un grupo de bajo peso y un grupo de obesidad mórbida. El estudio encontró que la disfunción sensorial se relaciona con la masa grasa, la masa libre de grasa, grasa visceral y varias adipocinas. Las evaluaciones del olfato fueron realizadas con la prueba de Sniffin' Sticks, y para determinar las capacidades gustativas se utilizó una prueba alemana llamada Taste Strips. Se encontró que las mujeres con obesidad tuvieron puntajes más bajos en las tres pruebas de olfato (umbral, discriminación e identificación). Las mujeres con obesidad también tuvieron menores puntajes en la prueba de percepción gustativa. La conclusión a la que se llegó es que existe una relación inversa entre grasa visceral y percepción de señales olfatorias y gustativas (FernándezGarcía et al., 2017).

En una investigación reciente se compararon las respuestas de individuos con obesidad y con peso normal al olor del chocolate (Stafford \& Whittle, 2015). Se encontró que los individuos con obesidad percibían el olor a chocolate como más placentero y fueron sustancialmente más sensibles a este estímulo. Los hallazgos apoyan la hipótesis de que los individuos con obesidad muestran una sensibilidad aumentada y mayor preferencia por olores asociados con alimentos altos en calorías. Los autores sugieren que los grupos de personas con obesidad se caracterizan por un aumento en la percepción sólo a los olores de los alimentos, pero no por otro tipo de olores (Stafford \& Welbeck, 2011; Stafford \& Whittle, 2015).

En relación con esto último, también se ha reportado que los individuos con obesidad perciben los estímulos gustativos dulces más intensamente que los individuos con peso normal (Bartoshuk et al., 2006). Es de particular interés mencionar que en una investigación realizada por Trellakis y colaboradores (Trellakis et al., 2011) se encontró que el olor de la pimienta negra es percibido como menos placentero por individuos con obesidad en comparación con sujetos con peso normal. Una posible explicación para este hallazgo es que la asociación de un olor a los alimentos de sabor amargo (generalmente de menor valor calórico) es más negativa en individuos con obesidad que en individuos sin obesidad.

Parece posible que, dadas las diferencias sensoriales entre individuos con obesidad y con peso normal, las alteraciones en el procesamiento de la información olfatoria asociada a los alimentos facilitan la sobreingesta en sujetos con obesidad. Un estudio de resonancia magnética funcional, estudió la respuesta de las regiones cerebrales a la estimulación con odorantes asociados a alimentos en individuos con obesidad y con peso normal, enfocándose en regiones involucradas en el procesamiento del valor de la recompensa de un estímulo. Los resultados indican que los estímulos odorantes asociados a los alimentos son procesados por el cerebro de modo distinto en comparación con estímulos no asociados a alimentos, y que el peso corporal se asocia a cambios en la activación de regiones cerebrales que determinan el valor de una recompensa por medio de la interpretación de señales olfatorias (Eiler et al., 2014).

Por otro lado, aunque los estudios mencionados asumen que el efecto olfatorio que se observa en la obesidad es un rasgo, sólo recientemente la comunidad científica se ha preguntado si ese efecto pudiera tratarse de un estado. Un ensayo en humanos reveló que un ayuno de 24 horas puede inducir un incremento en la capacidad olfatoria (Cameron, Goldfield \& Doucet, 2012). Por el contrario, una dieta hiperlipidémica causa una disminución de esta modalidad sensorial a largo plazo, como se ha observado en ratones (Thiebaud et al., 2014). En esta misma línea, Takase, Tsuneoka, Oda, Kuroda \& Funato (2016) mostraron que ratones con peso normal pero alimentados con una dieta alta en grasa, requerían el mismo tiempo, que ratones con obesidad, para encontrar galletas de maní cubiertas bajo tierra. Estos autores concluyeron que una dieta alta en grasa afecta el olfato independientemente de la obesidad. Se espera que estos hallazgos sean confirmados en el futuro cercano. 


\section{Conclusiones}

La evidencia actual señala que el sentido del olfato está alterado en la población con obesidad. Es necesario investigar si se trata de una disminución generalizada, como lo indican algunos estudios, o si la percepción está aumentada para algunos estímulos (p. ej. estímulos de alto valor calórico) y disminuida para otros (p. ej. estímulos no asociados con alimentos). También es preciso averiguar cuáles son los mecanismos involucrados en las alteraciones de la percepción olfatoria en personas con obesidad. El estudio del sentido del olfato es un área prometedora para la investigación de la obesidad.

\section{Agradecimientos}

Marco Guarneros recibió una beca posdoctoral del Consejo Nacional de Ciencia y Tecnología(CVU:227789) en el programa Estancias Posdoctorales Nacionales.

\section{CONFLiCto DE INTERESES}

Los autores declaran que la investigación fue conducida en ausencia de relaciones comerciales o financieras que pudieran ser interpretadas como causa potencial de conflicto de interés.

\section{REFERENCIAS}

Apelbaum, A. F. \& Chaput, M. A. (2003). Rats habituated to chronic feeding restriction show a smaller increase in olfactory bulb reactivity compared to newly fasted rats. Chemical Senses, 28(5), 389-395. DOI: 10.1093/ chemse/28.5.389

Aschenbrenner, K., Hummel, C., Teszmer, K., Krone, F., Ishimaru, T., Seo, H. S. \& Hummel, T. (2008). The influence of olfactory loss on dietary behaviors. Laryngoscope, 118(1), 135-144. DOI: 10.1097/MLG.0b013e318155a4b9

Bartoshuk, L. M., Duffy, V. B., Hayes, J. E., Moskowitz, H. R. \& Snyder, D. J. (2006). Psychophysics of sweet and fat perception in obesity: problems, solutions and new perspectives. Philosophical Transactions of the Royal Society ofLondon. Series B, Biological Sciences, 361(1471), 1137-1148. DOI: 10.1098/rstb.2006.1853

Cameron, J. D., Goldfield, G. S. \& Doucet, É. (2012). Fasting for $24 \mathrm{~h}$ improves nasal chemosensory performance and food palatability in a related manner. Appetite, 58, 978-981. DOI: 10.1016/j.appet.2012.02.050

Donaldson, L. F., Bennet, L., Baic, S. \& Melichar, J. K. (2009). Taste and weight: is there a link? The American journal of clinical nutrition, 90(3), 800S-803S. DOI: 10.103945/ ajcn.2009.27462Q

Doty, R. L., Shaman, P., Kimmelman, C. P. \& Dann, M. S. (1984). University of Pennsylvania Smell identification test: a rapid quantitative olfactory function test for the clinic. Laryngoscope, 94(2 Pt. 1), 176-178. DOI: 10.1288/00005537-198402000-00004

Eiler, W. J.2 ${ }^{\text {nd }}$, Dzemidzic, M., Case, K. R., Armstrong, C. L., Mattes, R. D., Cyders, M. A., Considine, R.V. \& Kareken, D. A. (2014). Ventral frontal satiation-mediated responses to food aromas in obese and normal-weight women. American journal of clinical nutrition, 99(6), 1309-1318. DOI: $10.3945 /$ ajen.113.080788

FAO(Organización de las Naciones Unidas para laAlimentación y la Agricultura),, OPS (Organización Panamericana de la Salud)., WFP(Programa Mundial deAlimentos) \& UNICEF (Fondo de las Naciones Unidas para la Infancia). (2018). Panorama de la seguridad alimentaria y nutricional en América Latina y el Caribe 2018. Santiago, Chile. Disponible en: http://iris.paho.org/xmlui/handle/123456789/49616

Fernández-Aranda, F., Agüera, Z., Fernández-García, J. C., Garrido-Sánchez, L., Alcaide-Torres, J., Tinahones, F. J., Giner-Bartolomé, C., Baños, R. M., Botella, C., Cebolla, A., de la Torre, R., Fernández-Real, J. M., Ortega, F. J., Frühbeck, G., Gómez-Ambrosi, J., Granero, R., Islam, M. A., Jiménez-Murcia, S., Tárrega, S., Menchón, J. M., Fagundo, A. B., Sancho, C., Estivill, X., Treasure, J. \& Casanueva, F. F. (2016). Smell-taste dysfunctions in extreme weight/eating conditions: analysis of hormonal and psychological interactions. Endocrine, 51, 256-267. DOI: $10.1007 /$ s 12020-015-0684-9

Fernández-García J. C., Alcaide, J., Santiago-Fernández, C., Roca-Rodríguez, M. M., Aguera, Z., Baños, R., Botella, C., de la Torre, R., Fernández-Real, J. M., Fruhbeck, G., Gómez-Ambrosi, J., Jiménez-Murcia, S., Menchon, J. M., Casanueva, F. F., Fernández-Aranda, F., Tinahones, F. J. \& Garrido-Sánchez, L. (2017). An increase in visceral fat is associated with a decrease in the taste and olfactory capacity. PLoS ONE. 12:e0171204. DOI:10.1371/journal. pone. 0171204.

Firestein, S. (2001). How the olfactory system makes sense of scents. Nature, 413, 211. DOI: 10.1038/35093026

Giachetti, I., MacLeod, P. \& LeMagnen, J. (1970). [Influence of hunger and satiety states on responses of the olfactory bulb in rats]. Journal of physiology (Paris), 62 Suppl 2 (2), 280-281.

Gouveri, E., Katotomichelakis, M., Gouveris, H., Danielides, V., Maltezos, E. \& Papanas, N. (2014). Olfactory dysfunction in type 2 diabetes mellitus: an additional manifestation of microvascular disease? Angiology 65, 869-876. DOI: $10.1177 / 0003319714520956$

Guarneros, M., Hudson, R., López-Palacios, M. \& DruckerColín, R. (2015). Reference values of olfactory function for Mexico City inhabitants. Archives of medical research, 46(1), 84-90. DOI: 10.1016/j.arcmed.2014.12.005

Guarneros, M. \& Hudson R. (2016). Nariz: sensible víctima de la contaminación. Cienciay Desarrollo, Mayo-Junio, 54-59.

Hardikar, S., Höchenberger, R., Villringer, A. \& Ohla, K. (2017). Higher sensitivity to sweet and salty taste in obese compared to lean individuals. Appetite, 111, 158-165. DOI: 10.1016/j.appet.2016.12.017

Hendrikse, J., Cachia, R., Kother, E., McPhie, S., Skouteris, H. \& Hayden, M. (2015). Attentional biases for food cues in overweight and individuals with obesity: a systematic 
review of the literature. Obesity reviews, 16 (5), 424-432. DOI: $10.1111 /$ obr. 12265

Hummel, T. \& Nordin, S. (2005). Olfactory disorders and their consequences for quality of life. Acta Otolaryngologica, 125, 116-121. DOI: 10.1080/00016480410022787

Hummel, T., Sekinger, B., Wolf, S. R., Pauli, E. \& Kobal, G. (1997). 'Sniffin'Sticks': olfactory performance assessed by the combined testing odor identification, odor discrimination and olfactory threshold. Chemical senses, 22, 39-52. DOI: 10.1093/chemse/22.1.39

Islam, M. A., Fagundo, A. B., Arcelus, J., Agüera, Z., JiménezMurcia, S., Fenández-Real, J. M., Tinahones, F. J., de la Torre, R., Botella, C., Frühbeck, G., Casanueva, F. F., Menchón, J. M. \& Fenández-Aranda, F. (2015). Olfaction in eating disorders and abnormal eating behavior: a systematic review. Frontiers in psychology, 6, 1431. DOI: 10.3389/ fpsyg.2015.01431

Jung, J., Kim, D.I., Han, G. Y. \& Kwon, H.W. (2018). The effects of high fat diet-induced stress on olfactory sensitivity, behaviors, and transcriptional profiling in Drosophila melanogaster. International journal of molecular sciences, 19(10), 2855. DOI: 10.3390/ijms19102855

King, B. M. (2006). Amygdaloid lesion-induced obesity: relation to sexual behavior, olfaction, and the ventromedial hypothalamus. Americanjournal of physiology. Regulatory, integrative and comparative physiology, 291, R1201R1214. DOI: 10.1152/ajpregu.00199.2006

Liu, D., Archer, N., Duesing, K., Hannan, G. \& Keast, R. (2016). Mechanism of fat taste perception: association with diet and obesity. Progress in lipid research, 63, 41-49.DOI: 10.1016/j.plipres.2016.03.002

Lledo, P. M., Gheusi, G. \& Vincent, J. D. (2005). Information processing in the mammalian olfactory system. Physiological reviews, 85(1), 281-317. DOI: 10.1152/ physrev.00008.2004

Marin, C., Vilas, D., Langdon, C., Alobid, I., López-Chacón, M., Haehner, A., Hummel, T. \& Mullol, J. (2018). Olfactory dysfunction in neurodegenerative diseases. Currentallergy and asthma reports, 18(8), 42. DOI: $10.1007 /$ s11882-0180796-4

Obrebowski, A., Obrebowska-Karsznia, Z. \& Gawlinski, M. (2000). Smell and taste in children with simple obesity. Internationaljournal of pediatric otorhinolaryngology, 55, 191-196. DOI:10.1016/S0165-5876(00)00397-9

Overberg, J., Hummel, T., Krude, H. \& Wiegand, S. (2012). Differences in taste sensitivity between obese and nonobese children and adolescents. Archives of disease in childhood, 97(12), 1048-1052. DOI: 10.1136/ archdischild-2011-301189

Pager, J., Giachetti, I., Holley, A. \& Le Magnen, J. (1972). A selective control of olfactory bulb electrical activity in relation to food deprivation and satiety in rats. Physiology \& behavior, 9, 573-579. DOI: 10.1016/00319384(72)90014-5
Palouzier-Paulignan, B., Lacroix, M. C., Pascaline, A., Baly, C., Caillol, M., Congar, P., Julliard, K., Tucker, K. \& Fadool, D. A. (2012). Olfaction under metabolic influences. Chemical Senses, 37, 769-797. DOI: 10.1093/chemse/bjs059

Peng, M., Coutts, D., Wang, Y. \& Cakmak, Y .O. (2019). Systematic review of olfactory shifts related to obesity. Obesity reviews, 20(2), 325-338. DOI: 10.1111/obr.12800

Pepino, M. Y., Finkbeiner, S., Beauchamp, G. K. \& Mennella, J. A. (2010). Obese women have lower monosodium glutamate taste sensitivity and prefer higher concentrations than do normal-weight women. Obesity, 18(5), 959-965. DOI: $10.1038 /$ oby.2009.493

Popkin, B. M., Aldair, L. S. \& Ng, S. W. (2012). Global nutrition transition and the pandemic of obesity in developing countries. Nutrition reviews, 70(1), 3-21. DOI: 10.1111/j.1753-4887.2011.00456.x

Pruszewicz, A. (1965). Concerning the testing of the sense of smell and taste (in Polish). Otolaryngologia Polska, 19, 29-37.

Richardson, B. E., Vander Woude, E. A., Sudan, R., Thompson, J. S.\& Leopold, D. A. (2004). Altered olfactory acuity in the morbidly obese. Obesity surgery, 14, 967-969. DOI: 10.1381/0960892041719617

Riera C. E., Tsausidou, E., Halloran, J., Follet, P., Hahn, O., Pereira, M. M.A., Ruud, L.E., Alber, J., Tharp, K., Anderson, C. M., Brönneke, H., Hampel, B., Filho, C. D. M., Stahl, A., Brüning, J. C. \& Dillin, A. (2017). The sense of smell impacts metabolic health and obesity. Cell Metabolism, 26:198-211.e5. DOI: 10.1016/j.cmet.2017.06.015

Robinson, T. E. \& Berridge, K. C. (2008). The incentive sensitization theory of addiction: some current issues. Philosophical Transactions of the Royal Society B, 363, 3137-3146. DOI: 10.1098/rstb.2008.0093

Rogers, P. J., Burley, V .J.,Alikhanizadeh, L.A. \& Blundell, J. E. (1995). Postingestive inhibition of food intake by aspartame: importance of interval between aspartame administration and subsequent eating. Physiology \& Behavior. 1995. 57:489-493. DOI: 10.1016/0031-9384(94)00289-h

Rolls, E.T. (2011). Taste, olfactory and food texture reward processing in the brain and obesity. International Journal of Obesity, 35, 550-561. DOI: 10.1016/j. pneurobio.2015.03.002

Schriever, V. A., Agosin, E., Altundag, A., Avni, A., Cao Van, H., Cornejo, C., de los Santos, G., Fishman, G., Fragola, C., Guarneros, M., Gupta, N., Hudson, R., Kamel, R., Knaapila, A., Konstantinidis, I., Landis, B. N., Larsson, M., Lundström, J .N., Macchi, A., Mariño-Sánchez, F., Martinec Nováková, L., Mori, E., Mullol, J., Nord, M., Parma, V., Philpott, C., Propst, E. J., Rawan, A., Sandell, M., Sorokowska, A., Sorokowski, P., Sparing-Paschke, L .M., Stetzler, C., Valder, C., Vodicka, J. \& Hummel, T. (2018). Development of an international odor identification test for children: the Universal Sniff Test. Journal of Pediatrics, 198, 265-272. DOI: 10.1016/jpeds.2018.03.011. 
Simchen, U., Koebnick, C., Hoyer, S., Issanchou, S. \& Zunft, H. J. (2006). Odour and taste sensitivity is associated with body weight and extent of misreporting of body weight. European Journal of Clinical Nutrition, 60, 698-705. DOI: 10.1038/sj.ejcn.1602371

Skrandies, W. \& Zschieschang, R. (2015). Olfactory and gustatory functions and its relation to body weight. Physiology \& Behavior, 142, 1-4. DOI: 10.1016/j. physbeh.2015.01.024

Stafford, L. D. \& Welbeck, K. (2011). High hunger state increases olfactory sensitivity to neutral but not food odors. Chemical senses, 36, 189-198. DOI: 10.1093/chemse/bjq114

Stafford, L .D. \& Whittle, A. (2015). Obese individuals have higher preference and sensitivity to odor of chocolate. Chemical senses, 40(4), 279-284. DOI: 10.1093/chemse/ bjv007

Stevenson, R. J. (2010). An initial evaluation of the functions of human olfaction. Chemical senses, 35, 3-20. DOI: 10.1093/chemse/bjp083

Takase, K., Tsuneoka, Y., Oda, S., Kuroda, M. \& Funato, M. (2016) High-fat diet feeding alters olfactory-, social-, and reward-related behaviors of mice independent of obesity. Obesity, 24, 886 894. DOI: 10.1002/oby.21441

Thiebaud, N., Johnson, M. C., Butler, J. L., Bell, G. A., Ferguson, K. L., Fadool, A. R., Gale, A. M., Gale, D. S. \& Fadool, D. A. (2014). Hyperlipidemic diet causes loss of olfactory sensory neurons, reduces olfactory discrimination, and disrupts odor-reversal learning. Journal of neuroscience, 34, 6970-6984. DOI: 10.1523/ JNEUROSCI.3366-13-2014
Thomas-Danguin, T, Rouby, C, Sicard, G, Vigouroux, M, Johansson, A, Bengtzon, A, et al. (2001). Odours identification and discrimination: Influence of culture and typicality on performance. Chem Senses, 26, 1062.

Thomas-Danguin, T., Rouby, C., Sicard, G., Vigouroux, M., Farget, V., Johanson, A., Bengtzon, A., Hall, G., Ormel, W., De Graaf, C., Rousseau, F. \& Dumont, J. P. (2003). Development of the ETOC: a European test of olfactory capabilities. Rhinology, 41, 142-151.

Trellakis, S., Tagay, S., Fischer, C., Rydleuskaya, A., Scherag, A., Bruderek, K., Schlegl, S., Greve, J., Canbay, A. E., Lang, S. \& Brandau, S. (2011). Ghrelin, leptin and adiponectin as possible predictors of the hedonic value of odors. Regulatory peptides, 167, 112-117. DOI: 10.1016/j. regpep.2010.12.005

Tucker, R. M., Edlinger, C., Craig, B. A. \& Mattes, R. D. (2014). Associations between BMI and fat taste sensitivity in humans. Chemical senses, 39(4), 349-357. DOI: 10.1093/ chemse/bju006

Wang, E., Cakmak, Y. \& Peng, M. (2018). Eating with eyes - Comparing eye movements and food choices between overweight and lean individuals in a real-life buffet setting. Appetite, 125, 152-159. DOI: 10.1016/j.appet.2018.02.003

Yeomans, M.R. (2006). Olfactory influences on appetite and satiety in humans. Physiology \& behavior, 89(1), 10-14. DOI: 10.1016/j.physbeh.2006.04.010

Yokum, S., Ng, J. \& Stice, E. (2011). Attentional bias to food images associated with elevated weight and future weight gain: an fMRI study. Obesity, 19(9), 1775-1783. DOI: 10.1038/oby.2011.168 